\title{
Sequential Contraction Compression Devices Reduce Leg Circumference in Patients with Chronic Venous Insufficiency
}

Gimmelreich $\mathbf{D}^{1}$, Karsilnikov $\mathbf{V}^{2}$, Litman $\mathrm{L}^{3}$ and Rosenblum $\mathrm{J}^{4 *}$

${ }^{1}$ Director of Surgical Service, Clalit Health Services, Jerusalem, Israel

${ }^{2}$ Department of Vascular Surgery, Shaarei Zedek Medical Center, Jerusalem, Israel

${ }^{3}$ Department of Psychology, Lander College, New York, USA

${ }^{4}$ Diabetic Foot Service, Shaarei Zedek Medical Center, Jerusalem Israel

\section{Introduction}

The more advanced stages of venous disease, Chronic Venous Insufficiency (CVI) (C3-C6), appear to affect about 5\% of the population, with the prevalence of the end stages of CVI (active and healed venous ulcers, $\mathrm{C} 5+\mathrm{C} 6)$ estimated at $1-2 \%$ [1]. The mechanism of venous leg ulcer formation involves a sequence of pathophysiologic steps that are thought to include [1] reduction in venous outflow due to venous obstruction and/or reflux, [2] persistent venous hypertension, and subsequent increased capillary filtration and interstitial fluid load [3]. Typically, excess interstitial fluid is effectively removed by the lymphatics, but if the fluid load overwhelms the lymphatic capacity or if the lymphatics are defective, then the accumulation of interstitial fluid, macromolecules, and cytokines lead to edema (CEAP C 3 ), breakdown of subcutaneous tissue (C 4A,B ), and formation of ulcers (C 6 ) $[2,3]$. Thus in order to prevent development of venous leg ulcerations, treatment of the underlying venous insufficiency is imperative.

Compression of the leg is the mainstay of therapy in patients who have CVI and is based on the understanding that gradient compression helps to relieve symptoms and to improve venous function. A variety of compression devices are available, including elastic bandages, gradient compression stockings, and Intermittent Pneumatic Compression (IPC) Devices. This is because it has been shown that continued use of compression therapy leads to improvement in the symptoms and on signs of CVI and documentation of changes in venous blood flow velocities [4-6]. There are many problems with existing compression therapies; the most prominent being patient compliance [7].

Sequential Contraction Compression Device (SCCD) Therapy functions physiologically in much the same way as IPC's. The mechanism of action is very different however. Where IPC compresses the legs and the blood vessels with an external source, the SCCD generates the vascular compression by generating its own muscular contraction by electrical stimulation of the neuromuscular junction [8]. Further, the SCCD generates a series of electric impulses causing a peristaltic series of contractions in the leg. The contractions are timed so that they maximize the vascular response. SCCD like IPC has been shown to elevate blood flow during application both in healthy volunteers and in patients with a wide spectrum of vascular diseases [9].

The aim of this study was to evaluate the effect of SCCD with the Flowaid FA-100 SCCD device (Flowaid Medical Technologies Corp. New York, NY) in conditions of regular clinical use on patients with CVI. The authors also wanted to evaluate the safety of SCCD in patients with this condition.

\section{Methods}

This was an open label prospective study of one-month duration evaluating SCCD on patients with CVI. Fifteen patients with a diagnosis of CVI in both legs were evaluated. In Table 1 shows the patient demographics and their disease characteristics. Patients were included in the study if their treatment regimen included either elastic stockings or IPC therapy to both legs. Patients were required to prove that they understood the requirements of their participation and be willing to participate. Patients enrolled with informed consent. Patients were excluded if they were pregnant or nursing, or if there was an open ulcer in the area of the placement of the electrodes.

Patients were brought to the clinic and two lines were drawn around their legs in permanent marker, at the ankle and at the top third of the calf. They were placed in a sitting non-dependent position and given fifteen minutes to rest with their legs further elevated by a standard leg block. Leg circumference was taken at both markings on the legs. Patients were given therapy to the leg of their choice with the SCCD (Flowaid Medical Technologies Inc. New York, NY) for two hours while they were allowed to ambulate freely in the vicinity of the clinic. The contralateral leg was treated at the same time with either their elastic stockings or with their IPC device. If they were being treated with IPC they could not ambulate during the treatment. Patients were then brought back to the clinic and had their leg circumferences measured again.

\begin{tabular}{|c|c|c|c|c|c|c|c|c|}
\hline Patient & Sex & Age (yr) & C & E & A & P & C score & D score \\
\hline 1 & M & 51 & 5 & S & S,D,P & R & 8 & $2 / 3$ \\
\hline 2 & M & 59 & 6 & S & S,D,P & R & 10 & $2 / 3$ \\
\hline 3 & F & 70 & 6 & S & S,D,P & R & 11 & 2 \\
\hline 4 & M & 62 & 6 & S & S,D,P & R,O & 14 & 3 \\
\hline 5 & F & 60 & 5 & S & S,D,P & R,O & 10 & 3 \\
\hline 6 & M & 60 & 6 & S & S,D,P & R,O & 10 & 2 \\
\hline 7 & M & 75 & 6 & S & S,D,P & R & 12 & $2 / 3$ \\
\hline 8 & M & 50 & 6 & S & S,D,P & R & 10 & $2 / 3$ \\
\hline 9 & M & 75 & 6 & S & S,D,P & R & 10 & 3 \\
\hline 10 & F & 42 & 6 & S & S,D,P & R,O & 14 & 3 \\
\hline 11 & M & 61 & 5 & S & S,D,P & R & 9 & 2 \\
\hline 12 & F & 48 & 6 & S & S,D,P & R,O & 10 & $2 / 3$ \\
\hline 13 & F & 49 & 6 & S & S,D,P & R & 10 & 2 \\
\hline 14 & M & 48 & 5 & S & S,D,P & R & 11 & 3 \\
\hline 15 & F & 63 & 6 & S & S,D,P & R & 13 & $2 / 3$ \\
\hline
\end{tabular}

Table 1: Patient characteristics.

*Corresponding author: Rosenblum J, Director, Diabetic Foot Service Shaarei Zedek Medical Center, Jerusalem, Israel, Tel: +972 2-666-6666; E-mail: diabfootman@gmail.com

Received July 14, 2016; Accepted August 08, 2016; Published August 17, 2016

Citation: Gimmelreich D, Karsilnikov V, Litman L, Rosenblum J (2016) Sequential Contraction Compression Devices Reduce Leg Circumference in Patients with Chronic Venous Insufficiency. J Vasc Med Surg 4: 283. doi: 10.4172/23296925.1000283

Copyright: $\odot 2016$ Gimmelreich D, et al. This is an open-access article distributed under the terms of the Creative Commons Attribution License, which permits unrestricted use, distribution, and reproduction in any medium, provided the original author and source are credited. 


\begin{tabular}{|l|c|c|c|c|}
\hline Time & SCCD treated leg at the ankle $\mathbf{( c m )}$ & Contrlateral Leg at the ankle $\mathbf{( c m )}$ & SCCD treated leg at the calf $(\mathbf{c m})$ & Contralateral leg at the calf $(\mathbf{c m})$ \\
\hline Baseline & $25.1 \pm 0.6(19$ to 32.5$)$ & $24.9 \pm 0.7(18$ to 31.8$)$ & $39 \pm 1.6(29$ to 49.8 \\
\hline Two Hours & $22.9 \pm 1.2(16 \text { to } 31)^{\star}$ & $24.1 \pm 1.1(16$ to 33$)$ NS & $37.4 \pm 1.8(27$ to 48.1$)$ NS & $38.7 \pm 2.1(28.5$ to 50.1 NS \\
\hline 30 Days & $19.6 \pm 0.8(14 \text { to } 23.8)^{\star *}$ & $23.1 \pm 0.9(17 \text { to } 30.9)^{\star}$ & $31.4 \pm 1.5(24 \text { to } 33.2)^{\star *}$ & $37.1 \pm 2.3(26.8$ to 49.6$)$ NS \\
\hline
\end{tabular}

Table 2: Results of the study ( ${ }^{*}$ Represents $p<0.05{ }^{* *}$ Represents $p<0.01$ NS-Represents Non-significant) versus the baseline.

Patients were given the SCCD device to take home with them and were instructed to use it on the same limb for two hours twice daily. They were free to ambulate or perform any activities while using the SCCD. On the contralateral leg, patients were instructed to use either their elastic stockings or their IPC devices as they were previously instructed by their physicians. Patients were also asked to darken the line drawn on their leg during the month, if they noticed it getting light. Patients were brought back to the clinic after thirty days and had their leg circumference measured again. The study was approved by the IRB which deemed it low risk.

\section{Results}

In Table 2 shows the results for the measured leg circumferences across the study. The results were independent of age, duration of the condition, and circumference of the leg at presentation. There was $8.7 \%$ decrease in ankle circumference vs the baseline at 2 hours in the treated limb $(\mathrm{p}<0.05)$ and at thirty days there was a $21.9 \%$ decrease $(\mathrm{p}<0.01)$. Calf measurements decreased by $4.35 \%$ at two hours (non-significant) and by $19.49 \%$ at thirty days in the treated limb $(\mathrm{p}<0.01)$. In the legs that were treated with standard compression, there were no significant changes versus the baseline except at the ankle at thirty days, where there was a reduction of $7.23 \%(\mathrm{p}<0.05)$. The measurements for the ankle at two hours, and the calf at two hours and thirty days were $3.2 \%$, $-1.84 \%$ and $2.37 \%$ respectively.

There was a noticeable difference between the treated legs and the contralateral limb both at two hours and after thirty days of treatment. At two hours the difference was statistically $(p>0.05)$ non-significant between the groups at both the ankles and the calfs. At thirty days there was a statistically significant difference $(\mathrm{p}<0.01)$ at both the ankle and the calf.

Subjectively, patients reported feeling heavier in the leg treated with their previous device. Compliance with the SCCD was reported as $100 \%$ during the study. Patients reported $100 \%$ compliance with the treatment given to the contralateral leg during the duration of the study but reported that their usual compliance was on average $64 \%(50-80 \%)$.

\section{Discussion}

CVI is a severe medical problem which if left untreated progresses to complications including Venous Leg Ulcers (VLU). As the population ages, and with the more sedentary lifestyle and the prevalent obesity in the population, the incidence of CVI will continue to rise and become an even bigger burden on the healthcare budget. Compression therapy is the mainstay for maintenance as well as for positive therapeutic action. The problem with compression is the lack of patient compliance due to many factors, all of them inherent in the modalities themselves. The authors look at an alternative type of compression therapy which seeks to address and alleviate many of the factors contributing to the poor patient compliance.

Elastic stockings provide their benefit by compressing dilated superficial veins, thereby shunting blood into the deep system in a more "physiologically correct" fashion [10]. Mayberry et al. concluded that the beneficial effect of stockings was derived from compression of the superficial venous system and not from any change in deep venous hemodynamics [11].

The results of the study compared well with other results in the literature. Our results were better than those presented by Spence et al. who showed good results on patients with CVI with an inelastic stocking [12]. Part of this may be a result of the fact that our patient population all had chronic post phlebitis venous disease with severe calf muscle pump dysfunction. Ohgi et al. showed that no improvement was seen in patients who had a failed pump and were treated with standard compression [13]. Our results also compared favorably to those presented by Rasmussen et al. [14] although a large portion of their patient population was also varied.

The Flowaid FA-100 SCCD (Flowaid Medical Technologies Corp. New York, NY) is a self-contained electric muscle pump activator. It is user friendly, and does not limit patient activity at all during use. It is not difficult to apply and does not cause a feeling of overheating in the treated limb. For all of these reasons patient compliance is high. The device has three settings for contraction speed, that is set depending on the condition being treated and the condition of the vasculature in the treated leg [14]

While this study showed a good clinical effect, future research may want to include vascular hemodynamics studies in order to assess the underlying mechanism of SCCD. Also future research may want to evaluate if there is a difference between the settings on the SCCD.

\section{Conclusion}

While this was a small pilot study, and a randomized controlled trial is certainly necessary, the results indicate that SCCD is a good form of compression therapy and aids in the reduction of leg circumference in patients with CVI. Further research should focus on validating this prospectively and maximizing the SCCD's effect.

\section{References}

1. Graham ID, Harrison MB, Nelson EA, Lorimer K, Fisher A (2003) Prevalence of lower-limb ulceration: a systematic review of prevalence studies. Adv Skin Wound Care 16: 305-316.

2. Poeartsch H, Lee B (2014) Phlebology and lymphology - a family affair Phlebology 29: 645-647.

3. Mortimer PS (2000) Implications of the lymphatic system in CVI-associated edema. Angiology 51: 3-7.

4. Ledig, BL (1995) Classification and grading of chronic venous disease in the lower limbs: a consensus statement. Phlebology 10: 2766-2769.

5. Sarin S, Scurr JH, Smith PDC (1992) Mechanisms of action of externa compression on venous function. $\mathrm{Br} \mathrm{J}$ Surg 79: 499-502.

6. Keith SL, McLaughlin DJ, Anderson FA, Cardullo PA, Jones AE (1992) Do graduated compression stockings and pneumatic boots have an additive effect on the peak velocity of venous blood flow? Arch Surg 127: 727-730.

7. Sheldon RD, Roseguini BT, Laughlin MH, Newcomer SC (2013) New insights into the physiologic basis for intermittent pneumatic limb compression as a therapeutic strategy for peripheral artery disease. J Vasc Surg 58: 1688-1696.

8. Labropoulos N, Leon LR, Bhatti A, Melton S, Kang SS, et al. (2005) 
Citation: Gimmelreich D, Karsilnikov V, Litman L, Rosenblum J (2016) Sequential Contraction Compression Devices Reduce Leg Circumference in Patients with Chronic Venous Insufficiency. J Vasc Med Surg 4: 283. doi: 10.4172/2329-6925.1000283

Hemodynamic effects of intermittent pneumatic compression in patients with critical limb ischemia. J Vasc Surg 42: 710-716.

9. Rosenblum J, Gimmelreich D, Greenberg N (2016) Sequential Contraction Compression has a Positive Effect on Patients with Peripheral Arterial Disease. J Nov Physiother 6: 3.

10. O'Donnell JF Jr, Rosenthal DA, Callow AD, Ledig BL (1979) Effect of elastic compression on venous hemodynamics in post phlebitic limbs. JAMA 242: 2776-2778.

11. Mayberry JC, Moneta GL, Frang RD, Porter JM (1991) The influence of elastic compression stockings on deep venous hemodynamics. J Vasc Surg 13: 91-100.
12. Spence RK, Cahall E (1996) Inelastic versus elastic leg compression in chronic venous insufficiency: A comparison of limb size and venous hemodynamics. J Vasc Surg 24: 783-787.

13. Ohgi, S, Kanaoka Y, Mori T (1994) Objective evaluation of compression therapy for deep vein thrombosis by ambulatory strain-gauge plethysmography. Phlebology 9: 28-31.

14. Rasmussen JC, Aldrich MB, Tan IC, Darne C, Zhu B, et al. (2016) Lymphatic transport in patients with chronic venous insufficiency and venous leg ulcers following sequential pneumatic compression. J Vasc Surg 4: 9-17. 